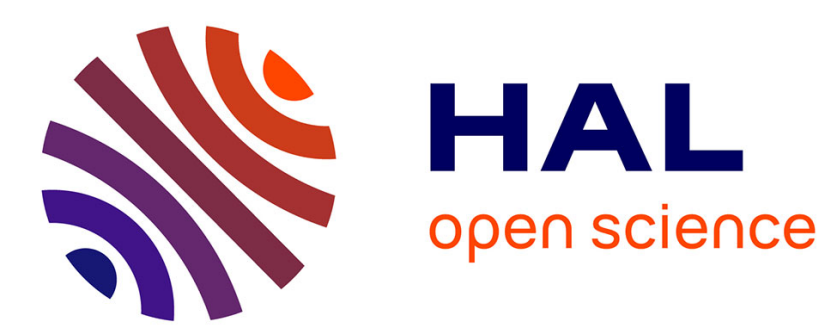

\title{
Regularization procedures of mixed finite element approximations of the Stokes problem
}

\author{
Roger Pierre
}

\section{To cite this version:}

Roger Pierre. Regularization procedures of mixed finite element approximations of the Stokes problem.

[Research Report] RR-0673, INRIA. 1987. inria-00075880

\section{HAL Id: inria-00075880 \\ https://hal.inria.fr/inria-00075880}

Submitted on 24 May 2006

HAL is a multi-disciplinary open access archive for the deposit and dissemination of scientific research documents, whether they are published or not. The documents may come from teaching and research institutions in France or abroad, or from public or private research centers.
L'archive ouverte pluridisciplinaire HAL, est destinée au dépôt et à la diffusion de documents scientifiques de niveau recherche, publiés ou non, émanant des établissements d'enseignement et de recherche français ou étrangers, des laboratoires publics ou privés. 


\section{Rapports de Recherche}

$$
\mathrm{N}^{\circ} 673
$$

\section{REGULARIZATION PROCEDURES OF MIXED FINITE ELEMENT APPROXIMATIONS OF THE STOKES PROBLEM}

Instititust National ded Recherche @ Informatique et en Automatique

Dornaine de Voluceau Rogquencourt B.P.105

78153 Le Chesnay Codex Frances

\section{Roger PIERRE}

\section{Mai 1987}




\section{Regularization procedures of mixed finite element approximations of the Stokes Problem}

\section{Méthodes de régularisation d'éléments mixtes pour l'approximation du Problème de Stokes* \\ Roger PIERRE}

Département de Mathématiques

Université Laval

Québec

G1K 7P4,Canada.

\section{Résumé}

Nous proposons un cadre théorique pour l'étude de la régularisation du problème de Stokes. Dans ce cadre, nous faisons une analyse d'erreur que nous appliquons à des schémas connus ainsi qu'à un nouveau schéma associé à l'élément P1-P1. Dans ce même cas, nous utilisons la théorie pour obtenir un résultat de convergence pour les éléments obtenus par addition de fonctions bulles, ceci sans avoir recours à la machinerie des éléments mixtes.

$\left({ }^{*}\right)$ : Ce travail a été réalisé au cours d'une année sabbatique durant laquelle l'auteur a séjourné au centre de Sophia-Antipolis invité par le projet Sinus. 


\section{Summary}

We propose a theoretical framework for the study of regularization of the Stokes problem. This enables us to perform a general error analysis and to apply it to known schemes as well as to a new one pertaining to the use of the P1-P1 element. Finally we show that in the P1-case the theory can also be used to get convergence results for elements obtained by addition of bubble functions, without using the usual mixed finite element machinery. 


\section{Introduction}

The approximation of the Stokes problem by Finite Element Methods has motivated a lot of research in the recent past. For the standard velocity- pressure formulation it was very early recognized that the approximation of the velocity field and of the pressure could not be chosen independently if one wanted to get a stable scheme. In particular equal-order $C^{0}$-interpolations are prohibited because they do not satisfy the classical inf-sup condition of Brezzi and Babǔska. On the other hand, since they are very attractive from the computational standpoint various approaches have been tried to modify the approximation setting in a way that would allow their use.

In [3], Brezzi and Pitkäranta studied two such approaches. The first one consists in the addition of the so called "bubble functions" to the discrete velocity space. The second amounts to modifying the discrete equations by the addition of a penalty-like term. This was generalized by Hughes and al. in [6]. There, they proposed a scheme very similar to the one of Pitkäranta but which is consistent. This idea of consistency appears to present both theoretical and practical advantages. The practical point of view was studied in [6] and [8], and the theoretical one, already considered in the aforementioned articles, will be developped here.

The purpose of this paper is twofold. First, we want to present the error analysis of a general regularizing scheme, showing, in particular, that, when consistency is preserved, it can lead to optimal $L^{2}$-rate of convergence. Next, we want to develop the relation observed in [8] between the use of regularization and of bubble functions for the P1-P1 element.

An outline of the remainder of the paper follows: In section 2, we review the fundamental facts about the continuous problem. In section 3 , we present the discretization that we want to study together with the necessary hypotheses on the mesh and the approximation spaces. The general error analysis is performed in section 4 and examples of consistent formulations are studied in section 5 . In section 6 , we look at the use of bubble functions and, in section 7 , we draw the conclusions.

\section{Formulation of the continuous problem}

Several Sobolev spaces will be used in the sequel. For their definition and the study of their various properties, see for example [7] or [5]. As usual, for a given space $H^{s}(D)$, the corresponding norm will be denoted by $\|\cdot\|_{a, D}$ and the semi-norm by $|\cdot|_{s, D}$ (in a clear context, we will drop the domain index).

We will also need the following : let

$$
H_{I R}^{s}(D)=\left\{g \in H^{s}(D) \mid \int_{D} g d \underline{x}=0\right\}
$$


(for $\mathrm{s}=0$ we use the standard notation $H_{\mathbb{R}}^{0}(D)=L_{0}^{2}(D)$ ), if we equip $H_{I R}^{s}(D)$ with the norm $\|\cdot\|_{\odot, D}$ it is isometric to the quotient space $H^{s}(D) / \mathbb{R}$ equipped with the usual quotient norm. In particular, on $H_{I R}^{1}(D)$, the norms $\|\cdot\|_{1, D}$ and $|\cdot|_{1, D}$ are equivalent.

Let $\Omega$ be an open bounded set of $\mathbb{R}^{N}, N=2,3$ with a regular boundary $\Gamma$. It will be practical for us to write the classical Stokes system in the general form:

$$
\left\{\begin{array}{l}
-\nu \Delta \underline{u}+\nabla p=\underline{f} \text { in } \Omega, \\
\nabla \cdot \underline{u}=g \text { in } \Omega, \\
\underline{u} \mid \mathbf{r}=0
\end{array}\right.
$$

From now on, we will, without loss of generality, set $\nu=1$ and select a fixed integer $m \geq 0$. We recall the following fundamental result, the proof of which can be found in [9].

Theorem A. Let $\Omega$ be an open set of $\mathbb{I R}^{N}$ of class $C^{r}, r=\max (m+2,2)$, and let $\underline{f} \in\left(H^{m}(\Omega)\right)^{2}, g \in H_{R}^{m+1}(\Omega)$ be given.Then (1) has a unique solution $(\underline{u}, p)$ in $\left(H^{m+2}(\Omega)\right)^{2} \times H_{I R}^{m+1}(\Omega)$. Moreover it satisfies

$$
\|\underline{\mathbf{u}}\|_{m+2}+\|p\|_{m+1} \leq C(m, \Omega)\left\{\|\underline{\mathbf{f}}\|_{m}+\|g\|_{m+1}\right\}
$$

It is also shown in [9], that $(\underline{u}, p)$ can be characterized as the unique solution of the following variational problem: Find $(\underline{u}, p) \in\left(H_{0}^{1}(\Omega)\right)^{2} \times L_{0}^{2}(\Omega)$ such that,

$$
\left\{\begin{array}{l}
\int_{\Omega} \nabla \underline{u}: \nabla \underline{v} d \underline{x}-\int_{\Omega} p \nabla . \underline{v} d \underline{x}=\int_{\Omega} \underline{f} . \underline{\mathrm{v}} d \underline{\mathrm{x}} \forall \underline{\mathrm{v}} \in\left(H_{0}^{1}(\Omega)\right)^{2} \\
\int_{\Omega} \nabla \underline{\underline{u}} q d \underline{\mathrm{x}}=\int_{\Omega} g q d \underline{\mathrm{x}} \quad \forall q \in L_{0}^{2}(\Omega)
\end{array}\right.
$$

Hereafter, we will refer to problem (3) as the continuous problem.

\section{Formulation of the discrete problem}

In this section we present a general approximation of (3), based on the idea of regularization as introduced in [3]. We limit ourselves to the case where $\Omega$ is polygonal and bounded in $\mathbb{R}^{2}$, but, in most cases, the results readily extend to $\Omega \subset \mathbb{R}^{3}$.

\subsection{Triangulation of $\Omega$. Properties of the discrete spaces}

Let $\left\{\tau_{h}\right\}_{h}$ be a family of regular triangulations of $\Omega$ such that $\bar{\Omega}=\bigcup_{K \in \tau_{h}} K$. We supnose that the elements are oither triangles or quadrilaterale and that each of 
them is affine equivalent to a reference element denoted by $\hat{K}$. We denote the diameter of $K$ by $h_{K}$ and set

$$
h=\max \left\{h_{K} \mid K \in \tau_{h}\right\}
$$

Apart from regularity, we make the following classical hypothesis: There exists a constant $c_{1, \tau}$ such that

$$
\forall h \quad \forall K \in \tau_{h} \quad h \leq c_{1, \tau} h_{K} .
$$

For each $h$, we associate to the triangulation $\tau_{h}$, two finite dimensional subspaces

$$
\begin{gathered}
V_{h} \subset\left(H_{0}^{1}(\Omega) \cap C^{0}(\bar{\Omega})\right)^{2}, \\
P_{h} \subset H_{I R}^{1}(\Omega) \cap C^{0}(\bar{\Omega}),
\end{gathered}
$$

and we assume that there exists two integers $k_{v}, k_{p}$ and two operators,

$$
\begin{aligned}
& \Pi_{v}:\left(H^{1}(\Omega)\right)^{2} \longrightarrow V_{h}, \\
& \Pi_{p}: H_{I R}^{1}(\Omega) \rightarrow P_{h},
\end{aligned}
$$

such that:

$$
\begin{gathered}
\forall 1 \leq k \leq k_{v}, \forall \underline{\mathrm{v}} \in\left(H^{k+1}(\Omega)\right)^{2}, \text { and } \forall 0 \leq l \leq k_{p}, \forall p \in H^{l+1}(\Omega), \\
\left|\underline{\mathrm{v}}-\Pi_{v}(\underline{\mathrm{v}})\right|_{0}+h\left|\underline{\mathrm{v}}-\Pi_{v}(\underline{\mathrm{v}})\right|_{1}+h^{2} \sum_{K \in \tau_{h}}\left|\underline{\mathrm{v}}-\Pi_{v}(\underline{\mathrm{v}})\right|_{2, K} \leq C h^{k+1}\|\underline{\mathrm{v}}\|_{k+1} \\
\left|p-\Pi_{p}(p)\right|_{0}+h\left|p-\Pi_{p}(p)\right|_{1} \leq C h^{l+1}\|p\|_{l+1} .
\end{gathered}
$$

We will also suppose that the following inverse inequalities are true (see [4]).

$$
\begin{gathered}
\forall \underline{\mathbf{v}}^{h} \in V_{h} \quad \forall K \in \tau_{h}, \quad\left|\underline{\mathbf{v}}^{h}\right|_{2, K} \leq c_{2, \tau} h^{-1}\left|\underline{\mathbf{v}}^{h}\right|_{1, K} \\
\forall q^{h} \in Q_{h} \quad \forall K \in \tau_{h}, \quad\left|q^{h}\right|_{1, \infty, K} \leq c_{3, \tau} h^{-N / 2}\left|q^{h}\right|_{1, K}
\end{gathered}
$$

Finally we will have use of a last notation. For $\underline{v} \in \prod_{K \in \tau_{h}}\left(H^{2}(K)\right)^{2}$ we write

$$
|\underline{\mathbf{v}}|_{j, h}=\left\{\sum_{K \in \tau_{h}}|\underline{\mathbf{v}}|_{j, K}^{2}\right\}^{\frac{1}{2}}, j=1,2
$$




\subsection{Regularized discretization procedures}

From now on, we will make the following hypotheses.

(H1): There exists a family of weight functions $\left\{w_{K}\right\}_{K \in \tau_{h}}$ with the following properties:

$$
\forall K \in \tau_{h}\left\{\begin{array}{l}
w_{K} \geq 0 \text { on } K, \\
w_{K} \in C^{0}(K) \text { and }\left\|w_{K}\right\|_{\infty, K} \leq c_{1, w} h^{2}, \\
\forall q^{h} \in Q_{h} \int_{K}\left(\nabla q^{h} \cdot \nabla q^{h}\right) w_{K} d \underline{x} \geq c_{2, w} h^{2}\left|q^{h}\right|_{1, K}^{2}
\end{array}\right.
$$

where $c_{1, w}$ and $c_{2, w}$ are strictly positive constants.

(H2): There exists a bilinear form $L_{h}\left(\underline{\mathrm{v}}, q^{h}\right)$ defined on $\prod_{K \in \tau_{h}}\left(H^{2}(K)\right)^{2} \times Q_{h}$ and which satisfies

$$
\left|L_{h}\left(\underline{\mathbf{v}}, q^{h}\right)\right| \leq\left|q^{h}\right|_{1}\left(c_{1, L} h|\underline{\mathbf{v}}|_{1, h}+c_{2, L} h^{2}|\underline{\mathbf{v}}|_{2, h}\right),
$$

where the $c_{i, L}$ are non-negative constants.

Using (7), we infer from (10) that, for each $\underline{\mathbf{v}}_{h} \in V_{h}$,

$$
\left|L_{h}\left(\underline{\mathrm{v}}^{h}, q^{h}\right)\right| \leq c_{L} h\left|q^{h}\right|_{1}\left|\underline{\mathbf{v}}^{h}\right|_{1}
$$

where $c_{L}=\max \left(c_{1, L}, c_{2, \tau} c_{2, L}\right)$. (Note that the case $c_{L}=0$ is allowed).

In the rest of the paper, we will study the following approximations of the Stokes problem :

Find $\left(\underline{u}^{h}, p^{h}\right) \in V_{h} \times P_{h}$ such that $\forall\left(\underline{v}^{h}, q^{h}\right) \in V_{h} \times P_{h}$,

$$
\left\{\begin{array}{l}
\int_{\Omega} \nabla \underline{\mathrm{u}}^{h}: \nabla \underline{\mathrm{v}}^{h} d \underline{\mathrm{x}}-\int_{\Omega} p^{h} \nabla \cdot \underline{\mathrm{v}}^{h} d \underline{\mathrm{x}}=\int_{\Omega} \underline{\mathrm{f}}^{\mathrm{v}} \underline{\underline{x}}^{h} d \underline{\mathrm{x}}, \\
\int_{\Omega} \nabla \cdot \underline{\mathrm{u}}^{h} q^{h} d \underline{\mathrm{x}}+\sum_{K \in \tau_{h}} \int_{K}\left(\left(\nabla \underline{p}^{h}-\underline{\mathrm{f}}\right) \cdot \nabla q^{h}\right) w_{K} d \underline{\mathrm{x}}-L_{h}\left(\underline{\mathrm{u}}^{h}, q^{h}\right)=\int_{\Omega} g q^{h} d \underline{\mathrm{x}} .
\end{array}\right.
$$

\section{Remark 1}

This form of regularization was suggested by the works of Brezzi and Pitkäranta [3] and of Hughes and al. [6]. The practical aspects of the implementation of such schemes have been considered in [8], where a relation between (12) and the use of bubble functions was pointed out. It is in the purpose of clarifying this last point that we introduced weight functions. It will be clear from the development below that we could replace, in (12), the weighted $L^{2}(K)$-inner product corresponding to $w_{K}$ by a more general bilinear form. We had no use of such a generality. As to the growth conditions imposed on $w_{K}$ and $L_{h}$, their justification will come from the error analysis. 


\section{Error analysis}

Before going to specific examples we present the error analysis of the general formulation (12).

\subsection{Existence and unicity of the discrete solution}

As expected, to obtain a general existence result, we have to impose conditions on the size of the operators appearing in (12). For this, set

$$
\forall \underline{\mathrm{v}}, \underline{\mathrm{u}} \in\left(H_{0}^{1}(\Omega)\right)^{2}, \quad a(\underline{\mathrm{v}}, \underline{\mathrm{u}})=\int_{\Omega} \nabla \underline{v}: \nabla \underline{u} d \underline{\mathrm{x}} .
$$

We recall the inequality

$$
a(\underline{u}, \underline{u}) \geq c_{\Omega}\|\underline{u}\|_{1} \quad \forall \underline{u} \in\left(H_{0}^{1}(\Omega)\right)^{2} .
$$

This leads us to

Theorem 1. Assume that, for a given $m \geq 0$, the regularity conditions of Theorem $A$ are fullfilled. If (H1) and (H2) are satisfied and if

$$
c_{2, w} c_{\Omega} \geq c_{L}^{2},
$$

is true, problem (12) has a unique solution in $V_{h} \times P_{h}$.

Proof. We set $W_{h}=V_{h} \times P_{h}$ and equip that space with the norm

$$
\left\|\left(\underline{\mathrm{v}}^{h}, q^{h}\right)\right\|_{W_{h}}^{2}=\left\|\underline{\mathrm{v}}^{h}\right\|_{1}^{2}+h^{2}\left|q^{h}\right|_{1}^{2} .
$$

Next we define on $W_{h}$, a bilinear form $A_{h}$ and a linear form $F_{h}$ by

$$
\begin{aligned}
& A_{h}\left(\left(\underline{\mathrm{v}}^{h}, q^{h}\right),\left(\underline{\mathrm{w}}^{h}, r^{h}\right)\right)=a\left(\underline{\mathrm{v}}^{h}, \underline{\mathrm{w}}^{h}\right)-\int_{\Omega} q^{h} \nabla \cdot \underline{\mathrm{w}}^{h} d \underline{\mathrm{x}} \\
& \quad+\int_{\Omega} \nabla \cdot \underline{\mathrm{v}}^{h} r^{h} d \underline{\mathrm{x}}+\sum_{K \in \tau_{h}}\left\{\int_{K}\left(\nabla q^{h} \cdot \nabla r^{h}\right) w_{K} d \underline{\mathrm{x}}\right\}-L_{h}\left(\underline{\mathrm{v}}^{h}, r^{h}\right), \\
& F_{h}\left(\left(\underline{\mathrm{v}}^{h}, q^{h}\right)\right)=\int_{\Omega} \underline{\mathrm{f}}^{h} \underline{\mathrm{v}}^{h} d \underline{\mathrm{x}}+\sum_{K \in \tau_{h}} \int_{K}\left(\underline{\mathrm{f}} \cdot \nabla q^{h}\right) w_{K} d \underline{\mathrm{x}}+\int_{\Omega} g q^{h} d \underline{\mathrm{x}} .
\end{aligned}
$$

Clearly (12) can be rewritten as: Find $\left(\underline{u}^{h}, p^{h}\right) \in W_{h}$ such that

$$
A_{h}\left(\left(\underline{\mathrm{u}}^{h}, \mathrm{p}^{h}\right),\left(\underline{\mathrm{v}}^{h}, q^{h}\right)\right)=F_{h}\left(\left(\underline{\mathrm{v}}^{h}, q^{h}\right)\right) \quad \forall\left(\underline{\mathrm{v}}^{h}, q^{h}\right) \in W_{h} .
$$


Since continuity is obvious, it follows from the Lax-Milgram lemma that the proof will be complete if we show that the form $A_{h}$ is $W_{h}$-elliptic.

For this, we use (9),(11) and (13) to obtain

$$
A_{h}\left(\left(\underline{\mathrm{v}}^{h}, q^{h}\right),\left(\underline{\mathrm{v}}^{h}, q^{h}\right)\right) \geq c_{\Omega}\left\|\underline{\mathrm{v}}^{h}\right\|_{1}^{2}+\sum_{K \in T_{h}}\left\{c_{2, w} h^{2}\left|q^{h}\right|_{1, K}^{2}\right\}-c_{L} h\left\|\underline{\mathrm{v}}^{h}\right\|_{1}\left|q^{h}\right|_{1}
$$

We deduce from the above that

$$
A_{h}\left(\left(\underline{\mathrm{v}}^{h}, q^{h}\right),\left(\underline{\mathrm{v}}^{h}, q^{h}\right)\right) \geq\left(\frac{c_{\Omega}}{2}\right)\left\|\underline{\mathrm{v}}^{h}\right\|_{1}^{2}+\left(c_{2, w}-\frac{c_{L}^{2}}{2 c_{\Omega}}\right) h^{2}\left|q^{h}\right|_{1}^{2} .
$$

Taking (14) into account, this readily leads to

$$
A_{h}\left(\left(\underline{\mathrm{v}}^{h}, q^{h}\right),\left(\underline{\mathrm{v}}^{h}, q^{h}\right)\right) \geq \frac{\min \left(c_{\Omega}, c_{2, w}\right)}{2}\left\|\left(\underline{\mathrm{v}}^{h}, q^{h}\right)\right\|_{W_{h}}^{2},
$$

which is is the desired result.

\subsection{Error estimates in the $H^{1}$-norm}

Looking at (12), it is easy to guess that the precision will depend both on the properties of $w_{K}$ and on the quality of the approximation of the laplacian term by $L_{h}$. To make this more precise, we introduce the following

Definition 1. Let $\underline{w}^{h}$ be in $V_{h}$, the consistency error on $\underline{w}^{h}$ is defined by

$$
E_{c}\left(\underline{\mathrm{w}}^{h}\right)=\sup _{P_{h} \backslash\{0\}} \frac{\left|e_{c}\left(\underline{w}^{h}, q^{h}\right)\right|}{\left|q^{h}\right|_{1}}
$$

where the consistency term $e_{c}\left(\underline{w}^{h}, q^{h}\right)$ is given by

$$
e_{c}\left(\underline{\mathrm{w}}^{h}, q^{h}\right)=\sum_{K \in T_{h}}\left[\int_{K}\left(\underline{\Delta u} \cdot \nabla q^{h}\right) w_{K} d \underline{\mathrm{x}}\right]-L_{h}\left(\underline{\mathrm{w}}^{h}, q^{h}\right)
$$

For a given $\underline{w}^{h}, e_{c}\left(\underline{w}^{h},.\right)$ is a linear operator on $P_{h}$, the norm of which is $E_{c}\left(\underline{w}^{h}\right)$. Moreover it satisfies,

$\forall \underline{w}^{h}, \forall \underline{r}^{h} \in V_{h}$,

$$
e_{c}\left(\underline{\mathrm{w}}^{h}+\underline{\mathrm{r}}^{h}, q^{h}\right)=e_{c}\left(\underline{\mathrm{w}}^{h}, q^{h}\right)-L_{h}\left(\underline{\mathrm{r}}^{h}, q^{h}\right) .
$$

The next Lemma justifies the introduction of that notioñ. 
Lemma 1. Assume that the regularity conditions of Theorem $A$ are fullfilled with $m \geq 0$ and that (H1),(H2) and the existence condition (14) are satisfied. Let $(\underline{u}, p)$ be the solution of the continuous problem and $\left(\underline{u}^{h}, p^{h}\right)$ be that of (12), we have: $\forall \underline{v}^{h} \in V_{h}, \forall q^{h} \in P_{h}:$

$$
\left\{\begin{array}{l}
a\left(\underline{\mathrm{u}}-\underline{\mathrm{u}}^{h}, \underline{\mathrm{v}}^{h}\right)-\int_{\Omega}\left(p-p^{h}\right) \nabla \cdot \underline{\mathrm{v}}^{h} d \underline{\mathrm{x}}=0, \\
\int_{\Omega} \nabla \cdot\left(\underline{\mathrm{u}}-\underline{\mathrm{u}}^{h}\right) q^{h} d \underline{\mathrm{x}}+\sum_{K \in T_{h}} \int_{K}\left[\nabla\left(p-p^{h}\right) \cdot \nabla q^{h}\right] w_{K} d \underline{\mathrm{x}}=e_{c}\left(\underline{\mathrm{u}}^{h}, q^{h}\right) .
\end{array}\right.
$$

Proof: In view of Theorem A, it directly follows from (1) and (H1) that (u, p) satisfies:

$\forall \underline{\mathrm{y}} \in\left(H_{0}^{1}(\Omega)\right)^{2}, \forall q \in L_{0}^{2}(\Omega)$

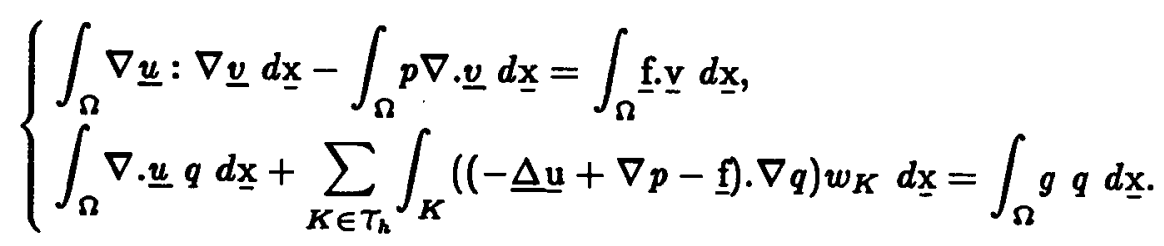

The result now readily follows by substracting (12) from (19).

This places us in position to state and prove the main result of this section.

Theorem 2. Let $1 \leq k \leq k_{v}$ and $0 \leq l \leq k_{p}$ be given. Under the hypotheses of Lemma 1, we have

$$
\left\|\underline{\mathrm{u}}-\underline{\mathrm{u}}^{h}\right\|_{1}+h\left|p-p^{h}\right|_{1} \leq c\left\{h^{k}\|\underline{\mathrm{u}}\|_{k+1}+h^{l+1}\|p\|_{l+1}+h^{-1} E_{c}\left(\Pi_{v} \underline{\mathrm{u}}\right)\right\} .
$$

Proof: In (18), we replace $\underline{u}-\underline{u}^{h}$ by $\underline{u}-\underline{w}^{h}+\underline{w}^{h}-\underline{u}^{h}$ and $p-p^{h}$ by $p-r^{h}+r^{h}-p^{h}$ where $\left(\underline{w}^{h}, r^{h}\right) \in V_{h} \times P_{h}$ is arbitrary. Next, we replace $\underline{\mathrm{v}}^{h}$ by $\underline{\mathrm{w}}^{h}-\underline{\mathrm{u}}^{h}$ and $q^{h}$ by $r^{h}-p^{h}$. Upon summing both equations side by side and using (17), we are lead to

$$
\begin{aligned}
& a\left(\underline{\mathrm{w}}^{h}-\underline{\mathrm{u}}^{h}, \underline{\mathrm{w}}^{h}-\underline{\mathrm{u}}^{h}\right)+\sum_{K \in \tau_{h}}\left[\int_{K}\left(\nabla\left(r^{h}-p^{h}\right) \cdot \nabla\left(r^{h}-p^{h}\right)\right) w_{K} d \underline{\mathrm{x}}=\right. \\
& a\left(\underline{\mathrm{w}}^{h}-\underline{\mathrm{u}}, \underline{\mathrm{w}}^{h}-\underline{\mathrm{u}}^{h}\right)-\int_{\Omega}\left(r^{h}-p\right) \nabla \cdot\left(\underline{\mathrm{w}}^{h}-\underline{\mathrm{u}}^{h}\right) d \underline{\mathrm{x}}+\int_{\Omega}\left(r^{h}-\underline{p}^{h}\right) \nabla \cdot\left(\underline{\mathrm{w}}^{h}-\underline{\mathrm{u}}\right) d \underline{\mathrm{x}} \\
& +\sum_{K \in \tau_{h}} \int_{K} \nabla\left(r^{h}-p\right) \cdot \nabla\left(r^{h}-p^{h}\right) w_{K} d \underline{\mathrm{x}}+e_{c}\left(\underline{\mathrm{w}}^{h}, r^{h}-p^{h}\right)-L_{h}\left(\underline{\mathrm{u}}^{h}-\underline{\mathrm{w}}^{h}, r^{h}-p^{h}\right) .
\end{aligned}
$$


We integrate the third term on the right by parts and use (13),(9),(16) and (11) to get

$$
\begin{aligned}
& c_{\Omega}\left\|\underline{\mathrm{w}}^{h}-\underline{\mathrm{u}}^{h}\right\|_{1}^{2}+c_{2, w} h^{2}\left|r^{h}-p^{h}\right|_{1}^{2} \leq\|a\|\left\|\underline{\mathrm{w}}^{h}-\underline{\mathrm{u}}\right\|_{1}\left\|\underline{\mathrm{w}}^{h}-\underline{\mathrm{u}}^{h}\right\|_{1} \\
& +\left|r^{h}-p\right|_{0}\left\|\underline{\mathrm{w}}^{h}-\underline{\mathrm{u}}^{h}\right\|_{1}+\left\|\underline{\mathrm{w}}^{h}-\underline{\mathrm{u}}\right\|_{0}\left|r^{h}-p^{h}\right|_{1} \\
& \quad+c_{1, w} h^{2}\left|r^{h}-p\right|_{1}\left|r^{h}-p^{h}\right|_{1}+E_{c}\left(\underline{\mathrm{w}}^{h}\right)\left|r^{h}-p^{h}\right|_{1} \\
& \quad+c_{L}\left\|\underline{\mathrm{u}}^{h}-\underline{\mathrm{w}}^{h}\right\|_{1}\left|r^{h}-p^{h}\right|_{1} .
\end{aligned}
$$

For each of the products appearing in the right-hand side, we use the arithmeticgeometric inequality to obtain,

$$
\begin{aligned}
c_{\Omega}\left\|\underline{w}^{h}-\underline{u}^{h}\right\|_{1}^{2}+c_{2, w} h^{2}\left|r^{h}-p^{h}\right|_{1}^{2} \leq \frac{\|a\|}{2}\left[\mu_{1}\left\|\underline{w}^{h}-\underline{u}^{h}\right\|_{1}^{2}+\frac{1}{\mu_{1}} \| \underline{w}^{h}-\underline{u}_{1}^{2}\right] \\
\quad+\frac{1}{2}\left[\mu_{2}\left|r^{h}-p\right|_{0}^{2}+\frac{1}{\mu_{2}}\left\|\underline{w}^{h}-\underline{u}^{h}\right\|_{1}^{2}\right]+\frac{1}{2}\left[\mu_{3}\left|r^{h}-p^{h}\right|_{1}^{2}+\frac{1}{\mu_{3}}\left\|\underline{w}^{h}-\underline{u}\right\|_{0}^{2}\right] \\
\quad+\frac{c_{1, w} h^{2}}{2}\left[\mu_{4}\left|r^{h}-p\right|_{1}^{2}+\frac{1}{\mu_{4}}\left|r^{h}-p^{h}\right|_{1}^{2}\right]+\frac{1}{2}\left[\mu_{5} E_{c}^{2}\left(\underline{w}^{h}\right)+\frac{1}{\mu_{5}}\left|r^{h}-p^{h}\right|_{1}^{2}\right] \\
\quad+\frac{1}{2}\left[c_{\Omega}\left\|\underline{u}^{h}-\underline{w}^{h}\right\|_{1}^{2}+\frac{h^{2} c_{L}^{2}}{c_{\Omega}}\left|r^{h}-p^{h}\right|_{1}^{2}\right],
\end{aligned}
$$

where the $\mu_{i}$ are to be determined. If we take (14) into account, and set

$$
c=\min \left(c_{\Omega}, c_{2, w}\right) \text {, }
$$

the choice

$$
\mu_{1}=\frac{c_{\Omega}}{4\|a\|}, \mu_{2}=\frac{4}{c_{\Omega}}, \mu_{3}=\frac{c_{2, w} h^{2}}{6}, \mu_{4}=\frac{6 c_{1, w}}{c_{2, w}} \text { and } \mu_{5}=\frac{6}{c_{2, w} h^{2}},
$$

leads to

$$
\begin{aligned}
& \frac{c}{4}\left\{\left\|\underline{\mathrm{w}}^{h}-\underline{\mathrm{u}}^{h}\right\|_{1}^{2}+h^{2}\left|r^{h}-p^{h}\right|_{1}^{2}\right\} \leq \frac{2\|a\|^{2}}{c}\left\|\underline{\mathrm{w}}^{h}-\underline{\mathrm{u}}\right\|_{1}^{2}+\frac{2}{c}\left|r^{h}-p\right|_{0}^{2} \\
& +\frac{3}{c h^{2}}\left\|\underline{\mathrm{w}}^{h}-\underline{\mathrm{u}}\right\|_{0}^{2}+\frac{3 c_{1, w}^{2} h^{2}}{c}\left|r^{h}-p\right|_{1}^{2}+\frac{3}{c h^{2}} E_{c}^{2}\left(\underline{\mathrm{w}}^{h}\right) .
\end{aligned}
$$

Now the necessity of the various growth conditions is clear.

To complete the proof, we first choose $\underline{w}^{h}=\Pi_{v}(\underline{u})$ and $r^{h}=\Pi_{p}(p)$, then we use the triangle in equality to obtain

$$
\begin{gathered}
\left\|\underline{\mathbf{u}}-\underline{\underline{u}}^{h}\right\|_{1}+h\left|p-p^{h}\right|_{1} \leq C\left\{h^{-1}\left[h\left\|\Pi_{v} \underline{\underline{u}}-\underline{\mathbf{u}}\right\|_{1}+\left\|\Pi_{v} \underline{u}-\underline{\mathbf{u}}\right\|_{0}\right]\right. \\
\left.\left.+\| \Pi_{p} p-\left.p\right|_{0}+h\left|\Pi_{p} p-p\right|_{1}\right]+h^{-1} E_{c}\left(\Pi_{v} \underline{u}\right)\right\},
\end{gathered}
$$

from which, taking (5) and (6) into account, the desired result follows.

In the application of Theorem 2, the crucial point will, in general, be the estimation of the last term in (20). As one may guess, this will require futher hypotheses. Before going to specific examples, we consider the problem of $L^{2}$-estimates. 


\subsection{Estimates in the $L^{2}$-norm}

Our approach is based on the classical Aubin-Nitsche argument and follows the lines of a similar proof given in [2].

Theorem 3. Under the hypotheses of Theorem 2, we have

$$
\left\|\underline{\mathbf{u}}-\underline{\underline{\mathbf{u}}}^{h}\right\|_{0}+h\left|p-p^{h}\right|_{0} \leq c\left\{h^{k+1}\|\underline{\mathbf{u}}\|_{k+1}+h^{l+2}\|p\|_{l+1}+E_{c}\left(\Pi_{v} \underline{\mathrm{u}}\right)\right\} .
$$

Proof: We first treat the case of the velocity. For this, consider the following auxiliary problem:

Find $(\underline{\eta}, \xi) \in\left(H_{0}^{1}(\Omega)\right)^{2} \times L_{0}^{2}(\Omega)$, such that

$$
\left\{\begin{array}{l}
a(\underline{\eta}, \underline{\alpha})-\int_{\Omega} \xi \nabla \cdot \underline{\eta} d \underline{x}=\int_{\Omega}\left(\underline{\underline{u}}-\underline{u}^{h}\right) \cdot \underline{\alpha} d \underline{x} \quad \forall \underline{\alpha} \in\left(H_{0}^{1}(\Omega)\right)^{2}, \\
\int_{\Omega} \nabla \cdot \underline{\eta} \zeta d \underline{x}=0 \quad \forall \zeta \in L_{0}^{2}(\Omega)
\end{array}\right.
$$

Since $m \geq 0$, it follows from Theorem A that problem (22) has a unique solution which verifies

$$
\|\underline{\eta}\|_{2}+|\xi|_{1} \leq c\left|\underline{u}-\underline{u}^{h}\right|_{0}
$$

Let us set $\underline{\alpha}=\underline{u}-\underline{u}^{h}$ in the first equation of (22). We obtain

$$
\left|\underline{u}-\underline{u}^{h}\right|_{0}^{2}=a\left(\underline{\eta}, \underline{u}-\underline{u}^{h}\right)-\int_{\Omega} \xi \nabla \cdot\left(\underline{u}-\underline{u}^{h}\right) d \underline{x},
$$

which we rewrite

$$
\begin{aligned}
\left|\underline{\mathrm{u}}-\underline{\mathrm{u}}^{h}\right|_{0}^{2}=a & \left(\underline{\eta}-\underline{\eta}^{h}, \underline{\mathrm{u}}-\underline{\mathrm{u}}^{h}\right)+a\left(\underline{\eta}^{h}, \underline{\mathrm{u}}-\underline{\mathrm{u}}^{h}\right) \\
& -\int_{\Omega}\left(\xi-\xi^{h}\right) \nabla \cdot\left(\underline{\mathrm{u}}-\underline{\mathrm{u}}^{h}\right) d \underline{\mathrm{x}}-\int_{\Omega} \xi^{h} \nabla \cdot\left(\underline{\mathrm{u}}-\underline{\mathrm{u}}^{h}\right) d \underline{\mathrm{x}},
\end{aligned}
$$

where $\underline{\eta}^{h}=\Pi_{v}(\underline{\eta})$ and $\xi^{h}=\Pi_{p}(\xi)$. We estimate the four terms of the right-hand side one by one. First,

$$
a\left(\underline{\eta}-\underline{\eta}^{h}, \underline{u}-\underline{u}^{h}\right) \leq\|a\|\left\|\underline{\eta}-\underline{\eta}^{h}\right\|_{1}\left\|\underline{u}-\underline{u}^{h}\right\|_{1} .
$$

Next, using Lemma 1 and the second equation in (22), we get

$$
a\left(\underline{\eta}^{h} ; \underline{u}-\underline{u}^{h}\right)=\int_{\Omega}\left(p-p^{h}\right) \nabla \cdot\left(\underline{\eta}^{h}-\underline{\eta}\right) d \underline{x},
$$


which, upon integrating by parts, leads to

$$
\left|a\left(\underline{\eta}^{h}, \underline{u}-\underline{u}^{h}\right)\right| \leq\left|p-p^{h}\right|_{1}\left|\underline{\eta}-\underline{\eta}^{h}\right|_{0}
$$

For the third term, we directly have

$$
\left|\int_{\Omega}\left(\xi-\xi^{h}\right) \nabla \cdot\left(\underline{u}-\underline{u}^{h}\right) d \underline{x}\right| \leq\left|\xi-\xi^{h}\right|_{0}\left\|\underline{u}-\underline{u}^{h}\right\|_{1} .
$$

The fourth term is the more troublesome. We use (18) to write it under the form

$$
\int_{\Omega} \nabla \cdot\left(\underline{u}-\underline{u}^{h}\right) \xi^{h} d \underline{x}=-\sum_{K \in \tau_{h}}\left\{\int_{K}\left[\nabla\left(p-p^{h}\right) \cdot \nabla \xi^{h}\right] w_{K} d \underline{x}\right\}+e_{c}\left(\underline{u}^{h}, \xi^{h}\right) .
$$

Using (9) and (16), we get as before

$$
\left|\int_{\Omega} \nabla \cdot\left(\underline{u}-\underline{u}^{h}\right) \xi^{h} d \underline{x}\right| \leq\left|\xi^{h}\right|_{1}\left\{c_{1, w} h^{2}\left|p-p^{h}\right|_{1}+E_{c}\left(\underline{u}^{h}\right)\right\}
$$

hence

$$
\left|\int_{\Omega} \nabla \cdot\left(\underline{u}-\underline{u}^{h}\right) \xi^{h} d \underline{x}\right| \leq\left\{c_{1, w} h^{2}\left|p-p^{h}\right|_{1}+E_{c}\left(\underline{u}^{h}\right)\right\}\left(\left|\xi^{h}-\xi\right|_{1}+|\xi|_{1}\right) .
$$

Grouping all the terms and going back to (24), we obtain, using (5),(6) and (23), that

$$
\left|\underline{u}-\underline{u}^{h}\right|_{0}^{2} \leq C\left|\underline{u}-\underline{u}^{h}\right|_{0}\left\{h\left\|\underline{u}-\underline{u}^{h}\right\|_{1}+h^{2}\left|p-p^{h}\right|_{1}+E_{c}\left(\underline{u}^{h}\right)\right\} .
$$

Notice that we are not yet done since it is the consistency error on $\Pi_{v} \underline{u}$ which we want on the right, not that on $\underline{u}^{h}$. Before taking care of that we turn to the case of the pressure. For this, change the dual problem to: Find $(\underline{\theta} \chi) \in\left(H_{0}^{1}(\Omega)\right)^{2} \times L_{0}^{2}(\Omega)$, such that

$$
\left\{\begin{array}{l}
a(\underline{\theta}, \underline{\alpha})-\int_{\Omega} \chi \nabla . \underline{\theta} d \underline{x}=0 \quad \forall \underline{\alpha} \in\left(H_{0}^{1}(\Omega)\right)^{2}, \\
\int_{\Omega} \nabla \cdot \underline{\theta} \varsigma d \underline{x}=\int_{\Omega}\left(p-p^{h}\right) \zeta d \underline{x} \quad \forall \varsigma \in L_{0}^{2}(\Omega)
\end{array}\right.
$$

Remark that, since both $p$ and $p^{h}$ where choosen in $H_{\mathbb{R}}^{1}$ the compatibility condition is fullfilled whence problem (30) has a unique solution which verifies

$$
\|\underline{\theta}\|_{2}+|\chi|_{1} \leq c\left|p-p^{h}\right|_{1} .
$$

As previously, taking $\underline{\theta}^{h}=\Pi_{v} \underline{\theta}$, we can write

$$
\left|p-p^{h}\right|_{0}^{2}=\int_{\Omega}\left(p-p^{h}\right) \nabla \cdot\left(\underline{\theta}-\underline{\theta}^{h}\right) d \underline{x}+\int_{\Omega}\left(p-p^{h}\right) \nabla \cdot \underline{\theta}^{h} d \underline{x} .
$$


The first term is directly estimated after integration by parts

$$
\left|\int_{\Omega}\left(p-p^{h}\right) \nabla \cdot\left(\underline{\theta}-\underline{\theta}^{h}\right) d \underline{x}\right| \leq\left|p-p^{h}\right|_{1}\left|\underline{\theta}-\underline{\theta}^{h}\right|_{0} .
$$

For the second term we go back to Lemma 1,

$$
\int_{\Omega}\left(p-p^{h}\right) \nabla \cdot \underline{\theta}^{h} d \underline{\mathrm{x}}=a\left(\underline{\mathrm{u}}-\underline{\mathrm{u}}^{h}, \underline{\theta}\right)-a\left(\underline{\mathrm{u}}-\underline{\mathrm{u}}^{h}, \underline{\theta}-\underline{\theta}^{h}\right) .
$$

Integrating the first term on the right by parts, which is correct since $\underline{\theta}$ is in $H^{2}$, we are lead to

$$
\left|\int_{\Omega}\left(p-p^{h}\right) \nabla \cdot \underline{\theta}^{h} d \underline{x}\right| \leq C\left\{\left|\underline{\mathbf{u}}-\underline{\underline{u}}^{h}\right|_{0}\|\underline{\theta}\|_{2}+\left\|\underline{\mathrm{u}}-\underline{\mathrm{u}}^{h}\right\|_{1}\left\|\underline{\theta}-\underline{\theta}^{h}\right\|_{1}\right\} .
$$

Using the last two in equalities with (32) and applying (5),(6) and (31) to $\underline{\theta}$, we get

$$
\begin{aligned}
& \quad\left|p-p^{h}\right|_{0}^{2} \leq \\
& \quad C\left\{h^{2}\left|p-p^{h}\right|_{1}^{2}+\left|\underline{u}-\underline{u}^{h}\right|_{0}\left|p-p^{h}\right|_{1}+h^{2}\left\|\underline{u}-\underline{u}^{h}\right\|_{1}\left|p-p^{h}\right|_{1}\right\} .
\end{aligned}
$$

We split the last two terms on the right as usual and obtain

$$
\left|p-p^{h}\right|_{0}^{2} \leq C\left\{h^{2}\left|p-p^{h}\right|_{1}^{2}+\left\|\underline{u}-\underline{u}^{h}\right\|_{1}^{2}+h^{-2}\left|\underline{u}-\underline{u}^{h}\right|_{0}^{2}\right\}
$$

Finally we combine (33) with (29),

$$
\left|\underline{\mathbf{u}}-\underline{\mathbf{u}}^{h}\right|_{0}+h\left|p-p^{h}\right|_{0} \leq C h\left\{\left\|\underline{\mathrm{u}}-\underline{\mathrm{u}}^{h}\right\|_{1}+h\left|p-p^{h}\right|_{1}+h^{-1} E_{c}\left(\underline{\mathrm{u}}^{h}\right)\right\} .
$$

To complete the proof, we must consider $E_{c}\left(\underline{u}^{h}\right)$. As noted before, we have

$$
e_{c}\left(\underline{u}^{h}, q^{h}\right)=e_{c}\left(\Pi_{v} \underline{u}, q^{h}\right)-L_{h}\left(\underline{u}^{h}-\Pi_{v} \underline{u}, q^{h}\right),
$$

hence, we infer from (16) and (11) that

$$
\begin{aligned}
\left|E_{c}\left(\underline{\underline{u}}^{h}\right)\right| & \leq\left|E_{c}\left(\Pi_{v} \underline{u}\right)\right|+c_{L} h\left|\underline{u}^{h}-\Pi_{v} \underline{u}\right|_{1} \\
& \leq\left|E_{c}\left(\Pi_{v} \underline{u}\right)\right|+c_{L} h\left|\underline{u}-\Pi_{v} \underline{u}\right|_{1}+c_{L} h\left|\underline{u}-\underline{u}^{h}\right|_{1} .
\end{aligned}
$$

Using this, we can rewrite (34) as

$$
\begin{aligned}
& \left|\underline{\mathbf{u}}-\underline{\underline{u}}^{h}\right|_{0}+h\left|p-p^{h}\right|_{0} \leq \\
& \quad C\left\{h\left\|\underline{\mathbf{u}}-\underline{\underline{u}}^{h}\right\|_{1}+h^{2}\left|p-p^{h}\right|_{1}+h\left|\underline{\mathrm{u}}-\Pi_{v} \underline{\mathrm{u}}\right|_{1}+E_{c}\left(\Pi_{v} \underline{\mathrm{u}}\right)\right\}
\end{aligned}
$$

and the result follows from Theorem 1 and (5). 


\section{Construction of consistent schemes}

In this section, we study a first approach to the construction of regularized formulations. In all the examples, we take the weight functions to be constant and concentrate on the approximation of the laplacian term.

\subsection{Definition and error estimate}

Definition 2. A regularization scheme of the type under consideration will be called consistent if, with $\underline{u}$ denoting the velocity solution,

$$
L_{h}\left(\underline{u}, q^{h}\right)=\sum_{K \in \tau_{h}} \int_{K}\left(\underline{\Delta} \underline{u} \cdot \nabla q^{h}\right) w_{K} d \underline{x}, \quad \forall q^{h} \in P_{h} .
$$

The interest of that notion is demonstrated by the following corollary.

Corollary 1. Let $1 \leq k \leq k_{v}$ and $0 \leq l \leq k_{p}$ be given. Under the hypotheses of Lemma 1 , if the formulation (12) is consistent, we have the estimate

$$
\begin{aligned}
\left\|\underline{\mathrm{u}}-\underline{\underline{u}}^{h}\right\|_{1}+h\left|p-p^{h}\right|_{1} \leq C\left\{h^{k}\|\underline{u}\|_{k+1}+h^{l+1}\|p\|_{l+1}\right\} \\
\left|\underline{\mathrm{u}}-\underline{\underline{u}}^{h}\right|_{0}+h\left|p-p^{h}\right|_{0} \leq C\left\{h^{k+1}\|\underline{\mathrm{u}}\|_{k+1}+h^{l+2}\|p\|_{l+1}\right\}
\end{aligned}
$$

Proof: In view of (35), we have

$$
e_{c}\left(\Pi_{v} \underline{u}, q^{h}\right)=L_{h}\left(\underline{u}-\Pi_{v} \underline{u}, q^{h}\right),
$$

from which, using (10) and (5), we infer that

$$
\begin{aligned}
E_{c}\left(\Pi_{v} \underline{\mathrm{u}}\right) & \leq c_{1, L} h\left|\underline{\mathrm{u}}-\Pi_{v} \underline{\mathrm{u}}\right|_{1}+c_{2, L} h^{2}\left|\underline{\mathrm{u}}-\Pi_{v} \underline{\underline{u}}\right|_{2, h} \\
& \leq C h^{k+1}\|\underline{\mathrm{u}}\|_{k+1} .
\end{aligned}
$$

The result is now a direct consequence of Theorem 2 and 3.

\subsection{The consistent scheme of Hughes and al.}

In [6], Hughes and al. proposed the following choice:

$$
\begin{aligned}
w_{K} & =\alpha h_{K}^{2} \quad \forall K \in \tau_{h}, \\
L_{h}\left(\underline{\mathbf{v}}, q^{h}\right) & =\sum_{K \in \tau_{h}} \alpha h_{K}^{2} \int_{K} \underline{\Delta} \underline{u} \cdot \nabla q^{h} d \underline{x} .
\end{aligned}
$$


where $\alpha$ is an ajustable positive parameter.

Quite obviously, the hypotheses (H1) and (H2) are satisfied and the corresponding formulation is consistent.

To check the validity of Corollary 1 , the only thing that we must do is to determine the choice of $\alpha$ for which the existence condition is fullfilled. For this note that

$$
c_{2, w}=\frac{\alpha}{c_{1, \tau}^{2}} \text { and } c_{L}=\alpha c_{2, \tau}
$$

thus (14) reads in this case

$$
\alpha \leq \frac{c_{\Omega}}{c_{1, \tau}^{2} c_{2, \tau}^{2}} .
$$

When this inequality is satisfied Corollary 1 is true, as was already proved in [6] for the $H^{1}$ case and in [2] for the $L^{2}$ one.

\section{Remark 2.}

Although the above formulation is always consistent in the sense defined here, for low order elements such as the P1-P1 or even the Q1-Q1 on a rectangular mesh the term $L_{h}\left(\underline{\mathrm{v}}^{h}, q^{h}\right)$ is identically zero, hence its introduction in (12) is somewhat artificial. In [8], we have shown that this absence of a velocity regularizing term had some consequences on the boundary behaviour of the discrete pressure and proposed two alternate formulations as remedy.

\subsection{Another approximation of the laplacian}

Let $\mathrm{N}=2$. We recall the following relation which is valid for any $H^{2}$ function.

$$
-\underline{\Delta \mathbf{v}}=\underline{\operatorname{rot}}(\operatorname{rot} \underline{\mathbf{u}})-\nabla(\nabla \cdot \underline{\mathbf{v}}),
$$

where, for $\underline{u}=\left(u_{1}, u_{2}\right)$, rot $\underline{u}=\frac{\partial u_{2}}{\partial x}-\frac{\partial u_{1}}{\partial y}$, whereas for any $\phi, \underline{\operatorname{rot}}(\phi)=\left(\frac{\partial \phi}{\partial y},-\frac{\partial \phi}{\partial x}\right)$. This suggests the choice

$$
\begin{aligned}
L_{h}\left(\underline{\mathrm{v}}, q^{h}\right) & =-\sum_{K \in \tau_{h}} \alpha h_{K}^{2} \int_{K} \underline{\operatorname{rot}}(\operatorname{rot} \underline{\mathrm{u}}) \cdot \nabla q^{h} d \underline{\mathbf{x}}, \\
w_{K} & =\alpha h_{K}^{2} \quad \forall K \in \tau_{h} .
\end{aligned}
$$

Using (39) and the fact that $\nabla . \underline{u}=0$, we see that this formulation is again consistent. The other verifications are identical to those made above and, under condition (38), Corollary 1 is valid.

As such, this idea is of no use in the P1-P1 case but for higher order elements it provides a non vanishing velocity regularizing term. 


\subsection{Regularization through a boundary integral}

To apply Hughes's idea in the P1-P1 context, we look for an appropriate weak form of (39). To this end, notice that

$$
\forall q^{h} \in P_{h}, \nabla q^{h} \in H(\operatorname{rot}, \Omega)
$$

Hence, under the regularity conditions of Theorem $\mathbf{A}$ the following integration by parts is valid when $\underline{u}$ is the velocity solution.(see [5])

$$
\begin{aligned}
\sum_{K \in \tau_{h}} \int_{K} \underline{\Delta u} \cdot \nabla q^{h} d \underline{x} & =-\int_{\Omega} \underline{\operatorname{rot}}(\operatorname{rot} \underline{u}) \cdot \nabla q^{h} d \underline{\underline{x}} \\
& =\left\langle\operatorname{rot}(\underline{u}), \nabla q^{h} \cdot \tau\right\rangle_{\frac{1}{2},-\frac{1}{2}}
\end{aligned}
$$

where $\tau$ denotes the unit tangent to $\Gamma$ and the bracket the duality between the spaces $H^{\frac{1}{2}}(\Gamma)$ and $H^{-\frac{1}{2}}(\Gamma)$. On the other hand, since $q^{h} \in C^{\infty}(K) \quad \forall K \in \tau_{h}$, $\nabla q^{h} . \tau$ is in fact in $L^{2}(\Gamma)$ and we have

$$
\begin{aligned}
\sum_{K \in T_{h}} \int_{K} \underline{\Delta u} \cdot \nabla q^{h} d \underline{x} & =\int_{\Gamma} \operatorname{rot}(\underline{u}) \nabla q^{h} \cdot \tau d \sigma \\
& =\sum_{K \in T_{h}} \int_{\partial K \cap \Gamma} \operatorname{rot}(\underline{u}) \nabla q^{h} \cdot \tau d \sigma
\end{aligned}
$$

This motivates the following choice which provides a non-zero velocity regularizing term even for the P1-P1 element.

$$
\begin{aligned}
L_{h}\left(\underline{\mathrm{v}}, q^{h}\right) & =\alpha h^{2} \sum_{K \in \tau_{h}} \int_{\partial K \cap \Gamma} \operatorname{rot}(\underline{\mathrm{v}}) \nabla q^{h} \cdot \tau d \sigma, \\
w_{K} & =\alpha h^{2}, \quad \forall K \in \tau_{h} .
\end{aligned}
$$

Notice that, when $\underline{v} \in H^{2}(K),\left.\operatorname{rot}(\underline{v})\right|_{\Gamma} \in L^{2}(\partial K \cap \Gamma)$. Thus the above definition makes sense.

To verify the validity of Corollary 1 we need the following

Lemma 2. Let $K \in \tau_{h}$ be arbitrary. For $v \in H^{1}(K)$, we have,

$$
\|v\|_{L^{2}(\partial K)} \leq C\left(h^{-\frac{1}{2}}|v|_{0, K}+h^{\frac{1}{2}}|v|_{1, K}\right),
$$

where the constant $C$ is independent of $K$ and h. 
Proof: Let $F: \hat{K} \longrightarrow K$ be the affine transformation mapping $\hat{K}$ on to $K$, and $B$, its jacobian matrix. If, as usual, $v=F \circ \hat{v}$ the following inequality is easily checked

$$
\int_{\partial K}|v|^{2} d \sigma \leq\|B\| \int_{\partial \hat{K}}|\hat{v}|^{2} d \hat{\sigma} .
$$

On the other hand, if $v \in H^{1}(K), \hat{v} \in H^{1}(\hat{K})$ and

$$
\|\hat{v}\|_{L^{2}(\partial \hat{K})} \leq C(\hat{K})\left(|\hat{v}|_{0, \hat{K}}+|\hat{v}|_{1, \hat{K}}\right)
$$

Using the classical estimate (see [4]),

$$
|\hat{v}|_{j, \hat{K}} \leq C(j, N)\|B\|^{j}(|\operatorname{det} B|)^{-\frac{1}{2}}|v|_{j, K}, \quad j \geq 0,
$$

we get *

$$
\|v\|_{L^{2}(\partial K)} \leq C(N)\|B\|^{\frac{1}{2}}(|\operatorname{det} B|)^{-\frac{1}{2}}\left(|v|_{0, K}+\|B\||v|_{1, K}\right) .
$$

Finally, in view of the regularity assumption on the mesh, we have

$$
\|B\| \leq C h, \text { and, }|\operatorname{det} B| \leq \hat{C} h^{2},
$$

where the constants are independent of $h$. The conclusion now follows directly from inequality (41).

The consistency of the scheme under consideration follows from the motivation given above. The validity of (H1) is clear so we are left with the problem of checking (H2) and that of translating the existence condition.

Concerning (H2), we first remark that $\forall \underline{v} \in H^{2}(K), \forall q^{h} \in P_{h}$,

$$
\left|\int_{\partial K \cap \Gamma} \operatorname{rot}(\underline{\mathrm{v}}) \nabla q^{h} \cdot \tau d \sigma\right| \leq\|\operatorname{rot}(\underline{\mathrm{u}})\|_{L^{2}(\partial K)}\left\|\nabla q^{h}\right\|_{L^{2}(\partial K)} .
$$

The estimation of the first factor on the right is given by Lemma 2 whereas, for the second one, we can use the inverse inequality $(8)$, (we recall that $N=2$ ). Indeed we have

$$
\begin{aligned}
\left\|\nabla q^{h}\right\|_{L^{2}(\partial K)} & \leq h^{\frac{1}{2}}\left|q^{h}\right|_{1, \infty, K} \\
& \leq c_{3, \tau} h^{-\frac{1}{2}}\left|q^{h}\right|_{1, K}
\end{aligned}
$$

Combining the inequalities (42),(43) and (40) with the definition of $L_{h}$, we get

$$
\left|L_{h}\left(\underline{\mathrm{v}}, q^{h}\right)\right| \leq \alpha \tilde{C}\left|q^{h}\right|_{1}\left\{h|\underline{\mathrm{v}}|_{1, h}+h^{2}|\underline{\mathrm{v}}|_{2, h}\right\}
$$


where $\tilde{C}$ depends only on the regularity assumptions on the mesh. Hence (H2) is verified with $c_{1, L}=c_{2, L}=\alpha \tilde{C}$.

Finally, since $c_{2, w}=\alpha$, the existence condition (14) will be written

$$
\alpha \leq \frac{c_{\Omega}}{\tilde{c}_{L}^{2}}
$$

where $\tilde{c}_{L}=\tilde{C} \max \left(1, c_{2, \tau}\right)$. Thus, if $\alpha$ satisfies the above inequality, Corollary 1 is valid for the corresponding formulation (12).

\section{Remark 3.}

In the three cases that we have presented, the existence condition is giving an upper bound for the parameter $\alpha$. In practice, it was observed in [6] and [8] that, when the formulation under consideration was used with unstable elements such as the P1-P1 or the Q1-Q1, there was also a practical lower bound below which (12) would produce a pressure field polluted with oscillations.

\section{Regularization by means of weight functions}

We now want to consider the possibility of taking $L_{h}=0$ in (12). The practical advantage of this choice is that the system is symmetric which means, in particular, some memory saving.

\subsection{General error estimate}

The next Corollary is a direct consequence of Theorem 1 and 2

Corollary 2. Let $1 \leq k \leq k_{v}$ and $0 \leq l \leq k_{p}$ be given. If $L_{h} \equiv 0$ in (12) and if the hypotheses of Lemma 1 are satisfied, we have

$$
\begin{aligned}
& \left\|\underline{\mathrm{u}}-\underline{\mathrm{u}}^{h}\right\|_{1}+h\left|p-p^{h}\right|_{1} \leq C\left\{h^{k}\|\underline{\mathrm{u}}\|_{k+1}+h^{l+1}\|p\|_{l+1}+h|\underline{\mathrm{u}}|_{2}\right\} \\
& \left|\underline{\mathrm{u}}-\underline{\mathrm{u}}^{h}\right|_{0}+h\left|p-p^{h}\right|_{0} \leq C\left\{h^{k+1}\|\underline{\mathrm{u}}\|_{k+1}+h^{l+2}\|p\|_{l+1}+h^{2}|\underline{u}|_{2}\right\} .
\end{aligned}
$$

\section{Remark 4}

The simplest situation covered by Corollary 2 is the one studied by Brezzi and Pitkäranta in $[3]$, where

$$
\tilde{w}_{K}=\alpha i_{K}^{2}, \alpha>0 .
$$


It appears that the use of the corresponding formulation with high order element is not interesting since the consistency error would then be bigger than the interpolation one. On the other hand, it was observed in [8] that, in the P1-P1 and Q1-Q1 case, even if, with this choice of $L_{h}$ the existence condition (14) does not restrict the value of $\alpha$, the possibility of oscillations in the pressure field limits it from below whereas a boundary layer effect limits it from above. In fact, in general, the choice of the parameter is even more tricky here than in the case of consistent formulations.

\subsection{Regularization versus using bubble functions}

In this section we restrict our attention to the P1-P1 element. More precisely, we suppose that $\tau_{h}$ consists of triangles and that $V_{h}$ and $P_{h}$ are the corresponding $C^{0}$, $P 1$-finite element subspaces. We introduce a new hypothesis.

(H3): There exists a family of functions $\left\{\phi_{K}\right\}_{K \in \tau_{h}} \subset\left(H_{0}^{1}(\Omega) \cap C^{0}(\bar{\Omega})\right)^{2}$ such that,

(i) $\forall \underline{x} \in K \quad \phi_{K}(\underline{x}) \in[0,1]$,

(ii) $\forall \underline{x} \notin K^{\circ} \quad \phi_{K}(\underline{x})=0$,

(iii) There exists stricly positive constants $d_{i}, i=1,4$ independent of $h$, such that $\forall K \in \tau_{h}$,

$$
\begin{gathered}
d_{1} \leq\left|\phi_{K}\right|_{1, K} \leq d_{2}, \\
d_{3} h_{K}^{2} \leq m_{K}=\int_{K} \phi_{K} d \underline{x} \leq d_{4} h_{K}^{2} .
\end{gathered}
$$

Hereafter, the functions $\phi_{K}$ will be called generalized bubble functions.

We claim that, to each such family, we can associate a family of weight functions

$$
w_{K}=\frac{m_{K}}{\left|\phi_{K}\right|_{1, K}^{2}} \phi_{K}
$$

satisfying (H1). Indeed, the only problem would be to verify the third condition but it easily follows from (iii) if we recall that the functions $q^{h}$ are linear.

To the family $\left\{\phi_{K}\right\}_{K \in T_{h}}$ we associate the set

$$
\Phi_{h}=\left\{\phi_{K} \underline{\mathbf{v}} \mid \underline{\mathbf{v}} \in \mathbb{R}^{2}, K \in \tau_{h}\right\} .
$$

It follows from (H3) that $\Phi_{h}$ is a subspace of $\left(H_{0}^{1}(\Omega)\right)^{2}$ such that $\forall \underline{\mathbf{v}}^{h} \in V_{h}, \quad \forall \underline{\mathrm{w}}_{1}, \underline{\mathrm{w}}_{2} \in \mathbb{R}^{2}$ :

$$
\begin{aligned}
& a\left(\underline{\mathrm{v}}^{h}, \phi_{K} \underline{\mathrm{w}}_{1}\right)=0 \quad \forall K \in \tau_{h} .
\end{aligned}
$$

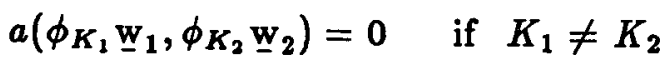


When $w_{K}$ is given by (44), we denote by $P_{h}$ the problem:

Find $\left(\underline{u}^{h}, p^{h}\right) \in V_{h} \times P_{h}$ such that $\forall\left(\underline{v}^{h}, q^{h}\right) \in V_{h} \times P_{h}$,

$$
\left\{\begin{array}{l}
a\left(\underline{u}^{h}, \underline{\mathrm{v}}^{h}\right)-\int_{\Omega} p^{h} \nabla \cdot \underline{\mathrm{v}}^{h}=\int_{\Omega} \underline{\mathrm{f}} \cdot \underline{\mathrm{v}}^{h} d \underline{\mathrm{x}}, \\
\int_{\Omega} \nabla \cdot \underline{\mathrm{u}}^{h} q^{h} d \underline{\mathrm{x}}+\sum_{K \in T_{h}} \int_{K}\left(\left(\nabla \underline{p}^{h}-\underline{\mathrm{f}}\right) \cdot \nabla q^{h}\right) w_{K} d \underline{\mathrm{x}}=\int_{\Omega} g q^{h} d \underline{\mathrm{x}} .
\end{array}\right.
$$

Similarly, if $\tilde{V}_{h}=V_{h} \oplus \Phi_{h}$, we denote by $\tilde{P}_{h}$ the problem:

Find $\left(\underline{\tilde{u}}^{h}, p^{h}\right) \in \tilde{V}_{h} \times P_{h}$, such that,

$$
\left\{\begin{array}{l}
a\left(\underline{\tilde{u}}^{h}, \underline{\tilde{\mathbf{v}}}^{h}\right)-\int_{\Omega} \boldsymbol{p}^{h} \nabla \cdot \underline{\tilde{\mathrm{v}}}^{h} d \underline{\mathrm{x}}=\int_{\Omega} \underline{\mathrm{f}} \underline{\tilde{\mathrm{v}}}^{h} \quad \forall \underline{\tilde{\mathrm{v}}}^{h} \in \tilde{V}_{h}, \\
\int_{\Omega} \nabla \cdot \underline{\tilde{u}}^{h} q^{h} d \underline{\mathrm{x}}=\int_{\Omega} g q^{h} d \underline{\mathrm{x}} \quad \forall q^{h} \in P_{h} .
\end{array}\right.
$$

The relation between $P_{h}$ and $\tilde{P}_{h}$ is illustrated in the following Lemma. Its proof can be obtained by combining the argument proposed in [8,section 3.4] with some elementary algebraic manipulations.

Lemma 3. Let $\left(\underline{u}^{h}, p^{h}\right) \in V_{h}$, and

$$
\underline{\tilde{u}}^{h}=\underline{u}_{h}+\sum_{K \in T_{h}} \phi_{K} \underline{u}_{K}
$$

where

$$
\underline{u}_{K}=\frac{1}{\left|\phi_{K}\right|_{1, K}^{2}} \int_{K}\left(\underline{f}-\nabla p^{h}\right) \phi_{K} d \underline{x},
$$

then, if (H3) is satisfied, $\left(\underline{u}^{h}, p^{h}\right)$ is a solution of $P_{h}$ if and only if $\left(\underline{\tilde{u}}^{h}, p^{h}\right)$ is a solution of $P_{h}$.

As shown by this Lemma, as far as one uses P1-elements, stabilization through the use of bubble functions is equivalent to a regularization of the type studied here. In particular one can hope that, for the elements obtained by addition of those generalized bubble functions, convergence results can be obtained from Corollary 2. This is confirmed by the next Theorem.

Theorem 4. Let $m=0$ in Theorem $A$. If (H3) is satisfied and if $\left(\underline{\tilde{u}}^{h}, p^{h}\right)$ is the solution of $\tilde{P}_{h}$ then

$$
\left\|\underline{\underline{u}}-\underline{\tilde{n}}^{h}\right\|_{\mathrm{i}}+\left|p-p^{h}\right|_{0} \leq C h\left(|\underline{\mathrm{f}}|_{0}+\|g\|_{1}\right) .
$$


Proof: The estimate for the pressure term follows directly from Corollary 2 and Theorem A. For the velocity term, write

$$
\left|\underline{u}-\underline{\tilde{u}}^{h}\right|_{1}^{2}=\left|\underline{u}-\underline{u}^{h}\right|_{1}^{2}-2 \sum_{K \in T_{h}} a\left(\underline{u}-\underline{u}^{h}, \phi_{K} \underline{u}_{K}\right)+\left|\sum_{K \in T_{h}} \phi_{K} \underline{u}_{K}\right|_{1}^{2} .
$$

Because of (45), we can estimate the second term in the following way,

$$
\left|\sum_{K \in \tau_{h}} a\left(\underline{u}-\underline{u}^{h}, \phi_{K} \underline{u}_{K}\right)\right| \leq|\underline{u}|_{2}\left\{\sum_{K \in \tau_{h}}\left|\phi_{K} \underline{u}_{K}\right|_{0, K}^{2}\right\}^{\frac{1}{2}}
$$

from which we deduce, using (i), that

$$
\left|2 \sum_{K \in \tau_{h}} a\left(\underline{u}-\underline{u}^{h}, \phi_{K} \underline{u}_{K}\right)\right| \leq C h|\underline{u}|_{2}\left\{\sum_{K \in \tau_{h}}\left\|\underline{u}_{K}\right\|_{\mathbb{R}^{2}}^{2}\right\}^{\frac{1}{2}} \text {. }
$$

Similarly, using (ii) and (45), we see that

$$
\left|\sum_{K \in T_{h}} \phi_{K} \underline{u}_{K}\right|_{1}^{2}=\sum_{K \in T_{h}}\left|\phi_{K}\right|_{1, K}^{2}\left\|\underline{u}_{K}\right\|_{I^{2}}^{2} \leq d_{2}^{2}\left(\sum_{K \in T_{h}}\left\|\underline{u}_{K}\right\|_{\mathbb{R}^{2}}^{2}\right) .
$$

On the other hand, using (46) and (i), we get

$$
\left\|\underline{u}_{K}\right\|_{\mathbb{R}^{2}} \leq \frac{1}{\left|\phi_{K}\right|_{1, K}^{2}}(\operatorname{meas}(K))^{\frac{1}{2}}\left|\underline{\mathrm{f}}-\nabla p^{h}\right|_{0, K},
$$

whence, using Corollary 2 with $k=1,1=0$ and Theorem $A$,

$$
\begin{aligned}
\left(\sum_{K \in T_{h}}\left\|\underline{\mathrm{u}}_{K}\right\|_{\mathbb{R}^{2}}^{2}\right)^{\frac{1}{2}} & \leq C h\left|\underline{\mathrm{f}}-\nabla p^{h}\right|_{0} \\
& \leq C h\left(|\underline{\mathrm{f}}|_{0}+\left|p-p^{h}\right|_{1}+|p|_{1}\right) \\
& \leq C h\left(|\underline{\mathrm{f}}|_{0}+\|g\|_{1}\right) .
\end{aligned}
$$

Finally, combining these inequalities, we deduce from (47) and Corollary 2 that

$$
\left|\underline{\mathbf{u}}-\underline{\tilde{u}}^{h}\right|_{1} \leq C h\left\{\left[\|\underline{\mathrm{u}}\|_{2}+\|p\|_{1}\right]+\|\left.\left.\underline{\mathrm{u}}\right|_{2}\left(|\underline{\mathrm{f}}|_{0}+\|g\|_{1}\right)\right|^{\frac{1}{2}}+\left(|\underline{\mathrm{f}}|_{0}+\|g\|_{1}\right)\right\}
$$

and the result follows from Theorem $A$.

It is easy to see that the corresponding $L^{2}$-estimate can be obtained through the Aubin-Nitsche argument as in Theorem 2. We will not work out the details but 
rather give two examples to which the theory applies.

Exemple 1: The Mini element.

This example was the motivation of the preceeding development. Let $\lambda_{i, K}, i=1,3$ denote the barycentric coordinates in the triangle $K$ and let

$$
\phi_{K}(\underline{x})= \begin{cases}27 \lambda_{1, K}(\underline{x}) \lambda_{2, K}(\underline{x}) \lambda_{3, K}(\underline{x}), & \text { for } \underline{x} \in K \\ 0, & \text { elsewhere }\end{cases}
$$

be the corresponding bubble function of degree 3 .

Using a different approach, Arnold, Brezzi and Fortin showed in [1] that, with this choice, the discretization $\tilde{P}_{h}$ was stable and that Theorem 4 was valid.

Let us look at this case from our point of view and check conditions (i)-(iii).

There is no problem for (i)-(ii). To check (iii), we recall that if $\phi_{\hat{K}}$ denote the bubble function associated with the reference element and if $B$ is the matrix of the affine transformation mapping $\hat{K}$ onto $K$, we have

$$
\left|\phi_{K}\right|_{1, K}^{2}=|\operatorname{det}(B)|\left|B^{-T} \nabla \phi_{\hat{K}}\right|_{0, \hat{K}}^{2}
$$

After some computations, we get

$\left|\phi_{K}\right|_{1, K}^{2}=\left(\frac{81}{20|\operatorname{det}(B)|}\right)\left\{\left(B_{2,2}^{2}+B_{1,2}^{2}\right)+\left(B_{1,1}^{2}+B_{2,1}^{2}\right)-\left(B_{2,1} B_{2,2}+B_{1,1} B_{1,2}\right)\right\}$.

If $\|\cdot\|_{F}$ denotes the Frobenius norm of a matrix, we deduce from the equivalence of norms on $\mathcal{L}\left(\mathbb{R}^{2}\right)$,

$$
C_{1} \frac{\|B\|^{2}}{|\operatorname{det}(B)|} \leq \hat{C}_{1} \frac{\|B\|_{F}^{2}}{|\operatorname{det}(B)|} \leq\left|\phi_{K}\right|_{1, K}^{2} \leq \hat{C}_{2} \frac{\|B\|_{F}^{2}}{|\operatorname{det}(B)|} \leq C_{2} \frac{\|B\|^{2}}{|\operatorname{det}(B)|}
$$

and the desired inequality follows from the regularity assumptions on the mesh. As to the condition on $m_{K}$, it follows at once from the equality

$$
m_{K}=\left(\frac{9}{40}\right)|\operatorname{det}(B)| \text {. }
$$

\section{Remark 5}

We have shown in [8] that, even though this element was converging at optimal rate, the pressure solution was often polluted by oscillations. The persistence of 
these oscillations was associated with the size of the coefficient of $w_{K}$ in (40).

\section{Exemple 2: Hat-functions.}

For a given triangle $K$, we denote the vertices by $\underline{b}_{i}, i=1,3$ and the barycenter by $\underline{\mathrm{b}}_{K}$. We also denote by $K_{i}, i=1,3$ the triangle obtained by joining $\underline{\mathrm{b}}_{K}$ to the two vertices $\underline{b}_{j}, j \neq i$.

With this, we define the following family of generalized bubble functions,

$$
\phi_{K}(\underline{x})= \begin{cases}3 \lambda_{i, K}(\underline{x}), & \text { if } \underline{x} \in K_{i} \\ 0, & \text { elsewhere }\end{cases}
$$

These functions are the familiar hat-functions. They certainly satisfy (i) and (ii). On the other hand, condition (iii) can be verified as above.

Indeed similar computations give

$$
\begin{gathered}
m_{K}=\left(\frac{1}{6|\operatorname{det}(B)|}\right), \\
\left|\phi_{K}\right|_{1, K}^{2}=\left(\frac{3}{|\operatorname{det}(B)|}\right)\left\{\|B\|_{F}^{2}+\left(B_{2,2} B_{2,1}+B_{1,2} B_{1,1}\right)\right\}
\end{gathered}
$$

whence the above reasonning can be applied.

\section{Remark 6}

Apart from presenting a different point of view on the study of those elements, the above theoretical approach provides us with a practical tool to predict their behaviour with respect to oscillations. Indeed, using (44) and the definitions, it is easy to deduce that

$$
\int_{K}\left(\nabla p^{h} \cdot \nabla q^{h}\right) w_{K} d \underline{x}=\left(\frac{m_{K}^{2}}{\operatorname{meas}(K)\left|\phi_{K}\right|_{1, K}^{2}}\right) \int_{K} \nabla p^{h} \cdot \nabla q^{h} d \underline{x} .
$$

In that form the pressure regularizing term is very similar to the one of Brezzi and Pitkäranta. In particular, as noted above, it appears that the bigger the coefficient of the right-hand side the better the smoothing. When we applied it to some regular grids, this criteria was always favoring the hat-functions over the bubble-functions. 


\section{Conclusion}

We have proposed a rather general framework for the study of regularized discretization of the Stokes equations which is covering all the examples known to the author. Within this framework, we have been able to get optimal error estimates in the $L^{2}$-norm for both the velocity and the pressure. In particular, this completes the study of a consistent scheme for the P1-P1 element introduced in [8].

Finally we have showed that this point of view can also be used to obtain a convergence result for the Mini element of Arnold, Brezzi and Fortin without resorting to the theory of saddle point problems.

\section{ACKNOWLEDGEMENTS}

This work was realized during a stay of the author at the INRIA-center of Sophia-Antipolis. He would like to take this opportunity to thank Professor Alain Dervieux for his kind invitation and for providing good facilities and a stimulating scientific environment.

\section{References}

1. Arnold D.N., F.Brezzi and M.Fortin, A stable finite element for the Stokes equations, to appear in Calcolo.

2. Brezzi F. and J. Douglas Jr., Stabilized mixed Methods, unpublished.

3. Brezzi F. and J.Pitkäranta, On the stabilization of finite element approximations of the Stokes equations, In: W.Hackbush(ed.), Efficients solutions of elliptic systems, Proceedings, Kiel,Jan. 1984. Notes on Numerical Fluid Mechanic,vol.10, pp 11-19, Vieweg, (1984).

4. Ciarlet P.G.: The finite element method for elliptic problems.Amsterdam: North Holland (1978).

5. Duvaut G. and J.L.Lions : Les inéquations en Mécaniques et en Physique. Dunod, Paris (1971).

6. Hughes T.J.R., L.P.Franca, M.Balestra, Circumventing the Babŭska-Brezzi condition: A stable Petrov-Galerkin Formulation of the Stokes Problem accomodating equal-order interpolation, preprint, September 1985, to appear in Computer Methods in Applied Mechanics and engineering.

7. Nečas J., Les méthodes directes en théorie des équations elliptiques, Masson, Paris (1967).

8. Pierre R., Simple $C^{0}$-approximations for the computations of incompressible flows, INRIA report (1987), submitted for publication.

9. Temam R., Navier-Stokes Equations, Amsterdam: North-Holland (1977). 
